\title{
A dynamic model of the
}

environmental Kuznets Curve:

turning point and public policy

\section{Journal Article}

\section{Author(s):}

Egli, Hannes; Steger, Thomas M.

Publication date:

2007-01

Permanent link:

https://doi.org/10.3929/ethz-b-000343236

Rights / license:

In Copyright - Non-Commercial Use Permitted

Originally published in:

Environmental and Resource Economics 36(1), https://doi.org/10.1007/s10640-006-9044-9 


\title{
A Dynamic Model of the Environmental Kuznets Curve: Turning Point and Public Policy
}

\author{
HANNES EGLI ${ }^{1, *}$ and THOMAS M. STEGER ${ }^{2}$ \\ ${ }^{1}$ CER-ETH - Center of Economic Research at ETH Zurich, ZUE F 10, CH-8092, Zurich, \\ Switzerland; ${ }^{2}$ CER-ETH - Center of Economic Research at ETH Zurich, ZUE F 13, \\ CH-8092, Zurich, Switzerland; *Author for correspondence (phone +41-44-632-0468; fax \\ +41-44-632-1362E-mail: hegli@ethz.ch)
}

Accepted 3 November 2005

\begin{abstract}
We set up a simple dynamic macroeconomic model with (i) polluting consumption and a preference for a clean environment, (ii) increasing returns in abatement giving rise to an EKC and (iii) sustained growth resulting from a linear final-output technology. There are two sorts of market failures caused by external effects associated with consumption and environmental effort. The model is employed to investigate the determinants of the turning point and the cost effectiveness of different public policies aimed at a reduction of the environmental burden. Moreover, the model offers a potential explanation of an N-shaped pollution-income relation. It is shown that the model is compatible with most empirical regularities on economic growth and the environment.
\end{abstract}

Key words: abatement, economic growth, environmental Kuznets curve, external effects, pollution, public policy

JEL classification: Q5, O4

\section{Introduction}

The Environmental Kuznets Curve (EKC) hypothesis states that there is an inverted U-shaped relationship between environmental degradation and the level of income. Starting with Grossman and Krueger (1993) this pattern has been intensively debated in empirical terms; recent reviews are provided by Dasgupta et al. (2002) and Stern (2004). The EKC has also captured considerable attention from policymakers and theorists. This is due to the fact that the EKC hypothesis implies that pollution diminishes once a critical threshold level of income is reached. As a consequence, there is the hope that loosely speaking - the environmental problem sooner or later peters out as the economy grows.

There are two major strands within the theoretical EKC literature. In the first class of models an EKC arises from shifts in the use of production technologies, which differ in their pollution intensity (Stokey 1998; Smulders et al. 2005). The second class focuses on the characteristics of the abatement 
technology (John and Pecchenino 1994; Selden and Song 1995; Andreoni and Levinson 2001; Chimeli and Braden 2002; Brock and Taylor 2004).

In an important paper, Andreoni and Levinson (2001) (thereafter AL) set up a static model to show that an EKC can be explained with increasing returns to scale (IRS) in the abatement technology. This approach can be viewed as a reduced form of a large number of models which focus on very different mechanisms (e.g. a shift in technology or a shift in institutions).

The level of income at which pollution peaks (labelled "the turning point") and the associated level of pollution are of fundamental interest from the perspective of public policy. A sound understanding of the pollutionincome relation (PIR) could provide important information for public policies aimed at a reduction of the environmental burden. The empirical EKC literature has accordingly devoted much effort to the determination of this critical threshold. The results show, however, a large dispersion across different studies. For instance, the reported turning points for sulphur dioxide range from $\$ 2,900$ to $\$ 908,200$ and for nitrogen oxides from $\$ 5,500$ to $\$ 30,800$ (in 1985 PPP\$; Lieb 2003). Given these diverse empirical results, it is clearly desirable to better understand the determinants of the turning point from a theoretical perspective.

We set up a simple dynamic EKC model with the following characteristics: Pollution is a by-product of consumption activities, it is modelled as flow pollution and it creates disutility. Households can spend resources on abatement to reduce gross pollution. Following AL we assume that there are IRS in abatement giving rise to an EKC. There are two market distortions due to external effects associated with consumption and abatement activities. Permanent growth results from an accumulable stock of capital and a linear final-output technology.

The paper at hand focuses on two issues: First, we employ the simple dynamic EKC model to better understand the determinants of the turning point. The factors which are of major interest in this type of models are the preference for a clean environment, the degree of IRS in abatement and the magnitude of external effects. Second, we investigate the effectiveness of public policy measures aimed at a reduction of the environmental burden. In this context, it is important to have a model with multiple market failures so that the question of relative policy effectiveness can be studied.

Pollution is modelled as flow pollution. The reason lies in the fact that an EKC is more likely to arise for flow pollutants than for stock pollutants. This is best illustrated by Lieb (2004, p. 484) who reports that "almost all studies agree that there is an EKC for sulphur dioxide $\left(\mathrm{SO}_{2}\right)$, suspended particulate matter (SPM), oxides of nitrogen $\left(N O_{x}\right)$, carbon monoxide $(C O)$, and for some (but not all) sorts of river pollution $(P R)$... Although all these pollutants 
are stock pollutants, they all have short life-times and can therefore be considered as flow pollutants from a long-run point of view."

There are a number of theoretical papers on the EKC which consider the determinants of the turning point; some of these papers also investigate the role of public policies. Brock and Taylor (2004) use an augmented Solow model to demonstrate that an EKC arises along the transition to the steady state. Although there is polluting production in this model, there is no market failure. Lieb (2004) uses an overlapping generations model with a stock pollution and a flow pollution. He focuses on the different pollution paths of the stock and the flow pollution. The model captures several external effects associated with production and abatement. However, only the problem of a myopic government is analysed implying that the intragenerational externalities are internalised, while the intergenerational externalities are not. Moreover, the effectiveness of public policy measures is not considered since the unregulated market economy is not investigated. Chimeli and Braden (2002) employ a simple endogenous growth model with environmental quality. They show that environmental quality follows a $\mathrm{V}$-shaped pattern, thereby explaining an EKC for a stock pollution. There is single external effect associated with polluting production. Hence, the consequences of multiple external effects cannot be studied. Finally, Anderson and Cavendish (2001) employ a dynamic simulation model to investigate the consequences of public policy measures on the turning point. This computable equilibrium model has the advantage of being able to directly include different aspects of the real world which are important in this context. However, general equilibrium feedback effects are excluded and optimal taxes cannot be derived.

In section 2, the basic AL model is sketched. In section 3, a simple dynamic EKC model is set up. The decentralised and the centralised solution are investigated and the optimal tax scheme is determined. In section 4 , a parameterised version of the model is employed to investigate the determinants of the turning point and the relative effectiveness of public policies. In section 5, it is shown that the model can potentially explain an $\mathrm{N}$-shaped PIR. Section 6 demonstrates that the model is compatible with important stylised facts on economic growth and the environment. Section 7 summarises and concludes.

\section{The Andreoni and Levinson EKC Model}

The AL (2001) model is sketched to provide a reference point for the following discussion. Utility of the representative agent depends positively on consumption $C$ and negatively on pollution $P$. The utility function is:

$$
U=U(C, P) .
$$


Pollution is a function of $C$ and environmental effort $E$ according to:

$$
P=C-B(C, E) .
$$

Pollution increases one-to-one with consumption (gross pollution), the first term on the RHS. On the other hand, pollution decreases due to abatement, the second term. The abatement technology $B(C, E)$ is increasing in both arguments. Both "inputs" are essential for abatement, i.e. $B(0, E)=B(C, 0)=0$. Finally, the resource constraint is $Y=C+E$, where $Y$ denotes available resources.

There are two conditions which together guarantee the existence of an EKC (AL 2001, p. 277). The first - related to preferences - states that "the marginal willingness to pay to clean up the last speck of pollution does not go to zero as income approaches infinity". This rather weak condition is easily satisfied since pollution abatement can be regarded as a normal good. ${ }^{1}$ The second condition - related to abatement technology - states that there must be IRS in abatement.

Using $U(C, P)=C-z P$ with $z=1$ and $B(C, E)=C^{\alpha} E^{\beta}$, AL show that an EKC results provided that $\alpha+\beta>1$. This can be seen by inspecting the pollution function in terms of $Y$ :

$$
P(Y)=\frac{\alpha}{\alpha+\beta} Y-\left(\frac{\alpha}{\alpha+\beta}\right)^{\alpha}\left(\frac{\beta}{\alpha+\beta}\right)^{\beta} Y^{\alpha+\beta} .
$$

The preceding equation results from $P=C-C^{\alpha} E^{\beta}, \quad C^{*}=\frac{\alpha}{\alpha+\beta} Y$ and $E^{*}=\frac{\beta}{\alpha+\beta} Y$, where $C^{*}$ and $E^{*}$ are the optimal levels of $C$ and $E$. Equation (3) implies that $P(Y)$ is concave in $Y$ provided that $\alpha+\beta>1$. Hence, IRS in abatement $(\alpha+\beta>1)$ represent a necessary condition for the existence of an EKC.

\section{A General Dynamic EKC Model}

A simple dynamic EKC model is set up. Pollution results as a by-product of consumption activities and is modelled as flow pollution. Households can reduce pollution by spending resources on abatement. The abatement technology is characterised by IRS, which gives rise to an EKC. There is a homogeneous final-output good which is produced under constant returns to scale using (physical and human) capital as the sole input factor. Households earn income by renting capital to firms. Output and factor markets are perfectly competitive. We consider two types of externalities and hence the decentralised solution diverges from the centralised solution. At first, the market economy is considered and then the centralised solution is investigated. Finally, the optimal tax scheme is determined. ${ }^{2}$ 


\subsection{THE DECENTRALISED ECONOMY}

There is a large number of identical households ordered on the interval $[0,1]$. The representative household derives utility from consumption $C$ and disutility from net pollution $P$. The instantaneous utility function is $U(C, P)$ with $U_{C}>0, U_{C C}<0, U_{P}<0$ and $U_{P P}<0 .{ }^{3}$ The flow of pollution (per period of time) is the difference between gross pollution $G(C, \bar{C})$ and abatement $B(C, E, \bar{E})$ :

$$
P(C, \bar{C}, E, \bar{E})=G(C, \bar{C})-\min \{B(C, E, \bar{E}), G(C, \bar{C})\},
$$

where $E$ is environmental effort and a "bar" above a variable denotes its economywide average. Pollution is modelled to result from consumption. ${ }^{4}$ Note that this definition implies that pollution cannot turn negative, which is appropriate for a pure flow pollution (Lieb 2004, p. 488; Egli 2005).

Direct examples for polluting consumption activities would be the use of automobiles and central heating. Turning to environmental effort, we can interpret the model in the sense that both households as well as firms conduct abatement. It is, however, plausible and convenient to let the incidence of abatement costs fall on households. To clarify this aspect, consider a real-world example: Abatement in the case of driving automobiles comprises the installation of catalytic converters and strainers. Although the major part of this abatement activity (development and installation) is conducted by firms, households face the decision for, and bear the costs of this environmental effort.

There are two kinds of externalities: First, polluting consumption is only partially taken into account by the representative household, i.e. there is a (negative) pollution externality. Second, environmental effort aimed at reducing (net) pollution affects also the society as a whole, i.e. there is a (positive) externality resulting from environmental effort. As an example, consider again the use of automobiles. It is the household who bears the financial burden but it is society that primarily benefits from the implementation of catalytic converters and strainers. External effects are associated with $\bar{C}$ and $\bar{E}$.

Let $r$ denote the rental price of capital $K$ owned by the representative household, who earns income of $r K$. Gross expenditures (including taxes) are given by $\left(1+\tau_{C}\right) C+\left(1+\tau_{E}\right) E$, where $\tau_{C}$ and $\tau_{E}$ represent taxes (subsidies) on $C$ and $E$. Tax revenues $T$ are redistributed in a lump-sum manner according to a balanced-budget rule, i.e. $T=\tau_{C} C+\tau_{E} E$. Households maximise the present value of an infinite utility stream. The associated dynamic problem may be expressed as (time index suppressed):

$$
\max _{\{C, E\}} \int_{0}^{\infty} U(C, P) e^{-\rho t} d t
$$




$$
\begin{array}{ll}
\text { s.t. } & P(C, \bar{C}, E, \bar{E})=G(C, \bar{C})-\min \{B(C, E, \bar{E}), G(C, \bar{C})\} \\
& \dot{K}=r K-\left(1+\tau_{C}\right) C-\left(1+\tau_{E}\right) E+T \\
& K(0)=K_{0},
\end{array}
$$

where $\rho$ is the time preference rate, $t$ the time index, $\dot{K}$ the rate of change of $K$ per period of time and $K_{0}$ the initial stock of capital, respectively.

The (current-value) Hamiltonian for this problem reads:

$$
H=U[C, P(C, \bar{C}, E, \bar{E})]+\lambda\left[r K-\left(1+\tau_{C}\right) C-\left(1+\tau_{E}\right) E+T\right],
$$

where $\lambda$ is the shadow price of $K$. The necessary conditions are given by: ${ }^{5}$

$$
\begin{aligned}
& \frac{U_{C}+U_{P} P_{C}}{1+\tau_{C}}=\lambda \\
& \frac{U_{P} P_{E}}{1+\tau_{E}}=\lambda \\
& \dot{\lambda}=-\lambda(r-\rho),
\end{aligned}
$$

where $U_{x}$ and $P_{x}$ denote the partial derivatives of $U$ and $P$ with respect to $x \in\{C, E\}$, respectively. For ease of interpretation, assume that $\tau_{C}=\tau_{E}=0$. Equation (10) then shows that along the optimal growth path the (private) marginal utility of consumption must equal $\lambda$. Marginal utility of consumption comprises two components: (i) direct utility from consumption $U_{C}$ and (ii) disutility from pollution $U_{P} P_{C}$. Remember that $P_{C}$ captures a gross pollution effect $G_{C}$ and an abatement effect $B_{C}$. Similarly, (11) indicates that marginal utility from environmental effort $U_{P} P_{E}$ must equal $\lambda$. Equation (12) shows that $\lambda$ vanishes at rate $r-\rho>0$.

The representative final-output firm produces a homogeneous good using capital only. The constant returns to scale technology is $Y=A K$, where $Y$ is final output and $A$ a constant technology parameter. Capital depreciates at constant rate $\delta \geq 0$. Output maximisation implies $r=A-\delta$.

\subsection{THE CENTRALISED ECONOMY}

The social planner maximises welfare of the representative individual, taking the external effects into account. The associated problem reads:

$$
\max _{\{C, \bar{C}, E, \bar{E}\}} \int_{0}^{\infty} U(C, P) e^{-\rho t} d t
$$




$$
\begin{array}{ll}
\text { s.t. } & P(C, \bar{C}, E, \bar{E})=G(C, \bar{C})-\min \{B(C, E, \bar{E}), G(C, \bar{C})\} \\
& \dot{K}=F(K)-\delta K-C-E \\
& K(0)=K_{0} .
\end{array}
$$

The (current-value) Hamiltonian reads:

$$
H=U[C, P(C, \bar{C}, E, \bar{E})]+\lambda[F(K)-\delta K-C-E]
$$

and the necessary conditions are given by: ${ }^{6}$

$$
\begin{aligned}
& U_{C}+U_{P}\left(P_{C}+P_{\bar{C}}\right)=\lambda \\
& U_{P}\left(P_{E}+P_{\bar{E}}\right)=\lambda \\
& \dot{\lambda}=-\lambda\left(F_{K}-\delta-\rho\right) .
\end{aligned}
$$

Comparing (18) and (19) to (10) and (11) shows the differences between the two solutions. When deciding on the optimal levels of $C$ and $E$ the social planner, in contrast to the private agent, takes the external consequences associated with $\bar{C}$ and $\bar{E}$ into account. Specifically, the social planner considers also the effects of $\bar{C}$ on gross pollution $\left(U_{P} P_{\bar{C}}=U_{P} G_{\bar{C}}\right)$ as well as the consequences of $\bar{E}$ on abatement $\left(U_{P} P_{\bar{E}}=-U_{P} B_{\bar{E}}\right)$.

\subsection{OPTIMAL TAX SCHEME}

Comparing (18) and (19) to (10) and (11) yields the optimal tax scheme:

$$
\begin{aligned}
\tau_{C}^{*} & =-\frac{U_{P} P_{\bar{C}}}{U_{C}+U_{P}\left(P_{C}+P_{\bar{C}}\right)}>0 \\
\tau_{E}^{*} & =-\frac{P_{\bar{E}}}{P_{E}+P_{\bar{E}}}<0 .
\end{aligned}
$$

Equation (22) shows that the optimal subsidy on environmental effort $\tau_{E}^{*}$ equals the ratio of the external marginal effect of environmental effort on pollution $P_{\bar{E}}<0$ and the overall (private and external) marginal effect of environmental effort on pollution $P_{E}+P_{\bar{E}}<0$. Similarly, the optimal consumption tax $\tau_{C}^{*}$ is the ratio of the external marginal consumption effect on utility $U_{P} P_{\bar{C}}<0$ and the overall marginal consumption effect on utility given by $U_{C}+U_{P}\left(P_{C}+P_{\bar{C}}\right)>0 .^{7}$ 
Consider the consequences of a consumption tax on the decisions of the representative household. Implementing $\tau_{C}>0$ reduces the LHS of (10). Holding $\lambda$ constant, (10) then requires the marginal utility of consumption to increase. This calls for a reduction of $C$. An analogous interpretation (with $\left.\tau_{E}<0\right)$ applies to (11).

\section{A Specific Dynamic EKC Model}

A parameterised version of the model is employed to investigate the determinants of the turning point and the effectiveness of public policies. At first, we consider the centralised solution. Subsequently, we turn to the more relevant case of an unregulated/imperfectly regulated economy.

\subsection{PARAMETERISATION}

We parameterise instantaneous utility $U(C, P)$, gross pollution $G(C, \bar{C})$ and abatement $B(C, E, \bar{E})$ as follows:

$$
\begin{aligned}
& U(C, P)=\log (C-z P) \quad \text { with } \quad z>0, C \geq z P \\
& G(C, \bar{C})=C^{1-\omega} \bar{C}^{\omega} \quad \text { with } \quad 0<\omega<1 \\
& B(C, E, \bar{E})=C^{\alpha} E^{\beta} \bar{E}^{\eta} \quad \text { with } \quad 0<\alpha, \beta, \eta<1,
\end{aligned}
$$

where $z$ shows the desire for a clean environment. A lower value of $z$ means that a given amount of pollution causes less disutility and individuals will accordingly spend more on $C$ and less on $E$. In (24) $C^{1-\omega}$ represents the internal effect of consumption on gross pollution and $\bar{C}^{\omega}$ is the corresponding external effect. Similarly, $E^{\beta}$ is the private and $\bar{E}^{\eta}$ the external effect of environmental effort in abatement. ${ }^{8}$

A short explanation of the instantaneous utility function (23) is indicated. Since $C=\bar{C}$ and $E=\bar{E}$, pollution is $P=C-C^{\alpha} E^{\beta+\eta}$. Moreover, assuming $z=1$ one gets $U[C, P(C, E)]=\log \left(C^{\alpha} E^{\beta+\eta}\right) .{ }^{9}$ This formulation has the advantage that $C$ and $E$ enter utility additively separable, which enables an analytical solution for the social planner's problem. 


\subsection{ANALYTICAL RESULTS}

The PIR is derived analytically and the determinants of the turning point are discussed. The focus is on the centralised solution with $z=1$, which allows derivation of analytical results.

\subsubsection{The time path of pollution $P(t)$ and the PIR $P(Y)$}

The model under study is an augmented AK-model which implies:

$$
\begin{aligned}
& K=K_{0} e^{(A-\delta-\rho) t} \\
& \lambda=\frac{\alpha+\beta+\eta}{K_{0} \rho} e^{-(A-\delta-\rho) t} .
\end{aligned}
$$

From (18), (19), (27) and (23) to (25) one gets:

$$
P(t)=\frac{K_{0} e^{(A-\delta-\rho) t} \alpha \rho}{\alpha+\beta+\eta}-\left(\frac{K_{0} e^{(A-\delta-\rho) t} \alpha \rho}{\alpha+\beta+\eta}\right)^{\alpha}\left(\frac{K_{0} e^{(A-\delta-\rho) t}(\beta+\eta) \rho}{\alpha+\beta+\eta}\right)^{\beta+\eta}
$$

Furthermore, the PIR may be expressed as follows:

$$
P(Y)=c Y-(c Y)^{\alpha}(h Y)^{\beta+\eta},
$$

where $c:=\frac{C}{Y}$ is the consumption rate and $h:=\frac{E}{Y}$ the "environmental effort rate". From $\hat{K}=A-\delta-\rho=A-\delta-C / K-E / K$ and the parameterised versions of (18) and (19) one gets:

$$
c=\frac{\alpha \rho}{A(\alpha+\beta+\eta)} \quad \text { and } \quad h=\frac{(\beta+\eta) \rho}{A(\alpha+\beta+\eta)} .
$$

The PIR is illustrated in Figure 1(a) and the time path of pollution in Figure 1(b). These graphs use the baseline set of parameters (section 3). As in AL (2001), IRS in abatement is a necessary condition for a hump-shaped PIR. ${ }^{10}$

Figure 1(a) shows that pollution first rises with income, then declines and eventually becomes zero. This EKC represents a balanced growth phenomenon. Although pollution does not grow at constant rate, the illustrated pollution path represents a balanced growth phenomenon since pollution results from two endogenous variables $(C$ and $E$ ), which both grow at constant rates. The required time span until pollution reaches its peak and 
becomes zero is quite long. The "EKC story" takes nearly 250 years, as displayed in Figure 1(b).

The EKC pattern displayed in Figure 1(a) is in line with empirical evidence reported by Grossman and Krueger (1995), which indicates that the PIR is asymmetric with an upper tail that declines relatively gradually.

\subsubsection{The turning point}

The determinants of the turning point are analysed. Closed-form solutions can only be obtained for the centralised economy with $z=1$. Under these restrictions one can investigate the impact of basic technology and preference parameters on the turning point analytically. This represents an interesting limiting case, which is relevant in the sense that the qualitative results largely hold true also for the decentralised economy with $z<1$, as investigated in section 4.3.

The point in time at which pollution reaches its maximum $\left(t^{*}\right)$ is:

$$
t^{*}=-\frac{\log \left[K_{0}^{\alpha+\beta+\eta-1} \alpha^{\alpha-1}(\beta+\eta)^{\beta+\eta}(\alpha+\beta+\eta)^{2-\alpha-\beta-\eta} \rho^{\alpha+\beta+\eta-1}\right]}{(\alpha+\beta+\eta-1)(A-\delta-\rho)} .
$$

Note that $z$ and $\omega$ do not appear on the RHS due to the restriction $z=1$ and the fact that $C=\bar{C}$. The turning point in terms of income $\left(Y^{*}\right)$ is: ${ }^{11}$

$$
Y^{*}=\frac{A \alpha^{\frac{1-\alpha}{\alpha+\beta+\eta-1}}(\beta+\eta)^{-\frac{\beta+\eta}{\alpha+\beta+\eta-1}}(\alpha+\beta+\eta)^{1-\frac{1}{\alpha+\beta+\eta-1}}}{\rho} .
$$

This critical income level is determined by the marginal product of capital $A$, the rate of time preference $\rho$, the elasticity of consumption in abatement $\alpha$ as well as the elasticities of environmental effort in abatement $\beta$ and $\eta$. It is independent of $\delta$ and $K_{0}$.

(a)

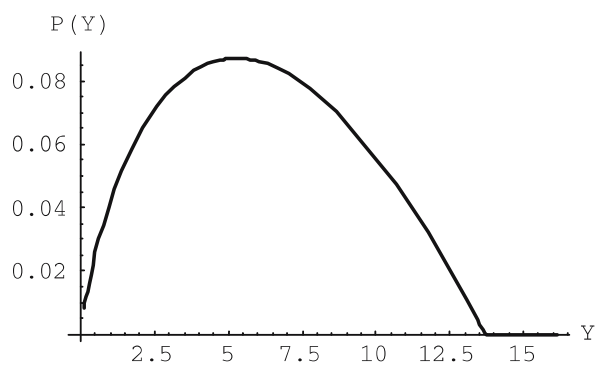

(b)

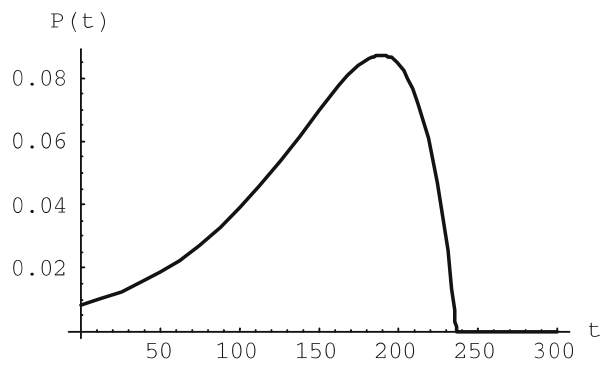

Figure 1. $P(Y)$ and $P(t)$ with IRS in abatement $(\alpha+\beta+\eta>1)$. 
Table I shows the comparative static results. ${ }^{12}$ The first row shows that $Y^{*}$ increases with $A$. For ease of interpretation, let us assume that $\alpha=\beta+\eta$ such that $C=E .^{13}$ In this case, the level of pollution depends only on consumption. Since an increase in $A$ reduces the consumption rate [see (30)], the required level of income for pollution to reach its maximum increases. The second row indicates that $Y^{*}$ falls as $\rho$ rises. An analogous reasoning is applicable here. The rate of consumption rises with $\rho$ [see (30)] and hence the required level of income for pollution to reach its maximum falls. The signs of the partial derivatives of $Y^{*}$ with respect to $\alpha$ and $\beta$ are indetermined. ${ }^{14}$ In most instances, the derivatives with respect to $\alpha$ and $\beta$ are negative. An increase in the degree of IRS in abatement leads, ceteris paribus, to a higher abatement output for each level of income and hence to a lower turning point. However, a positive sign cannot be excluded in general; for instance, under the restrictions $\alpha=\beta+\eta$ and $z=1$ the derivative with respect to $\alpha$ is positive. $^{15}$

\subsection{NUMERICAL ANALYSIS}

The preceding analysis focused on the centralised solution with $z=1$ implying that consumption and pollution have the same weight in the utility function. We now investigate the importance of external effects, the effectiveness of public policies and the implications of different environmental preferences, allowing for $z<1$. To accomplish this task, the transition process is simulated using the backward integration procedure (e.g. Brunner and Strulik 2002).

\subsubsection{Calibration}

Table II shows the employed baseline set of parameters. The time preference rate $\rho$ and the depreciation rate $\delta$ are similar to the parameter values used in previous exercises (e.g. Ortigueira and Santos 1997; Eicher and Turnovsky 2001). Given these values $A$ is chosen such that the implied net rate of return on capital $(A-\delta)$ and the growth rate of per capita income $(A-\delta-\rho)$ are in

Table I. Comparative static results for $Y^{*}$

\begin{tabular}{lll}
\hline & $\frac{\partial Y^{*}}{\partial x}$ for $x \in\{A, \rho, \alpha, \beta\}$ & \\
\hline$A$ & $Y^{*} \frac{1}{A}$ & $>0$ \\
$\rho$ & $Y^{*} \frac{-1}{\rho}$ & $<0$ \\
$\alpha$ & $Y^{*} \frac{(\gamma-1)(-\alpha+\beta+\eta)+\alpha \gamma(\log [\gamma]+(\beta+\eta)(\log [\beta+\eta]-\log [\alpha])}{\alpha \gamma(\gamma-1)^{2}}$ & $?$ \\
$\beta$ & $Y^{*} \frac{2+\gamma(\log [\gamma]-2)+\gamma(\alpha-1)(\log [\alpha]-\log [\beta+\eta])}{\gamma(\gamma-1)^{2}}$ & $?$ \\
\hline
\end{tabular}


line with empirically plausible numbers $(6 \%$ and $2 \%)$. We choose $\omega$ and $\eta$ such that the relative external effect of consumption in pollution $(\omega)$ and the relative external effect of environmental effort in abatement $\left(\frac{\eta}{\beta+\eta}\right)$ are both $10 \%$, implying fairly moderate external effects.

We assume $\alpha+\beta+\eta>1$. As in AL (2001), IRS in abatement are necessary for an EKC. This is in line with Xepapadeas (1994), where IRS in the pollution abatement sector (due to knowledge spillovers) is a necessary condition for unbounded growth without excess pollution (similarly Michel 1993). Alternatively, IRS in abatement may result from technological progress in the abatement technology (Anderson and Cavendish 2001). Regarding the empirical evidence, AL (2001, p. 281) argue that "at the level of US states, average pollution abatement costs per dollar of GSP [gross state product] decline with industry size, across states and industries, and over time." Maradan and Vassiliev (2005) report that the marginal opportunity costs of carbon dioxide abatement are negatively associated with income. Moreover, $\beta$ and $\eta$ crucially determine the ratio of abatement expenditures and income, which ranges from about $3 \%$ for $z=0.5$ to $15 \%$ for $z=1$. These values are in line with the empirical figures reported by Brock and Taylor (2004, p. 6).

\subsubsection{The turning point}

The dependence of $Y^{*}$ on the model parameters is investigated numerically. Three different values of $z$ are considered. In addition, the unregulated economy (Table III) is distinguished from an imperfectly regulated economy (Table IV). ${ }^{16}$ We focus on these two cases since we believe that the real world is best represented by an unregulated or imperfectly regulated economy. The basic assumption here is that politicians know the optimal taxes but due to imperfections in the political process do not fully implement optimal taxes. The numbers reported in Tables III and IV show the elasticities of $Y^{*}$ with respect to different model parameters, i.e. $\frac{\Delta Y^{*} / Y^{*}}{\Delta x / x}$ with $x \in\{\omega, \eta, A, \rho, \alpha, \beta, z\} .{ }^{17}$

Three points should be noted: First, the case of $z=1$ is qualitatively identical to the cases of $z<1$. By lowering $z$, the results change only gradually. The respective elasticities show the same sign for the unregulated economy (Table III) and for the imperfectly regulated economy (Table IV). Second, the analytical results from Table I are confirmed and the ambiguous

Table II. Baseline set of parameters

$\begin{array}{ll}\text { Final output technology } & A=0.12 ; \delta=0.06 \\ \text { Preferences } & \rho=0.04 \\ \text { Abatement technology } & \alpha=0.6 ; \beta=0.45 ; \eta=0.05 \\ \text { Gross pollution } & \omega=0.1\end{array}$


effects of $\alpha$ and $\beta$ are determined, at least numerically. Third, compared to the case investigated above (centralised solution with $z=1$ ) the impact of additional model parameters $(\omega$ and $\eta$ ) can be assessed.

The first column of Table III shows the elasticity of $Y^{*}$ with respect to $\omega$. The displayed positive impact can be explained as follows: Since the gross pollution function is linear, the level of centralised $C$ remains constant. Increasing $\omega$ leads to a larger gap between the centralised and the decentralised allocation. This implies that decentralised $C$ rises, which, ceteris paribus, causes a higher level of $P$ at each level of income. Graphically speaking, the EKC is expanded outwards and the turning point increases. This column also shows that the impact of $\omega$ on $Y^{*}$ increases with $z$. A higher value of $z$ (i.e. greener preferences) leads to a larger gap between the centralised and the decentralised solution, as can be seen by inspecting (18). This implies that the strength of the mechanism described above is reinforced. Finally, the effect of $\omega$ on $Y^{*}$ is smaller for the imperfectly regulated economy (Table IV).

The second column of Table III gives the impact of a variation in $\eta$ on $Y^{*}$, which is negative. An increase in $\eta$ has two separate effects: First, environmental effort falls. To understand this effect, consider the case of a variation in $\eta$ assuming that $\beta+\eta=$ constant. This implies that centralised $E$ remains constant. Since the magnitude of the distortion increases, the gap between the centralised and the decentralised solution gets larger. Hence, $E$ must decrease implying that pollution rises at each level of income and that the turning point increases as well. Second, by holding $\beta$ fixed (assumed in Tables III and IV), an increase in $\eta$ leads to a higher degree of IRS causing pollution to fall at each level

Table III. Elasticities of $Y^{*}$ with respect to model parameters; unregulated economy $(\theta=0)$

\begin{tabular}{llllllll}
\hline & $\omega$ & $\eta$ & $A$ & $\rho$ & $\alpha$ & $\beta$ & $z$ \\
\hline$Y^{*} z=1$ & 0.67 & -0.79 & 0.97 & -0.90 & -4.41 & -5.74 & -4.70 \\
$Y^{*} z=0.75$ & 0.46 & -1.45 & 0.98 & -0.90 & -7.48 & -7.40 & -4.42 \\
$Y^{*} z=0.5$ & 0.28 & -2.22 & 0.99 & -0.91 & -9.06 & -8.61 & -4.19 \\
\hline
\end{tabular}

Table $I V$. Elasticities of $Y^{*}$ with respect to model parameters; imperfectly regulated economy $(\theta=0.5)$

\begin{tabular}{llllllll}
\hline & $\omega$ & $\eta$ & $A$ & $\rho$ & $\alpha$ & $\beta$ & $z$ \\
\hline$Y^{*} z=1$ & 0.30 & -0.75 & 0.99 & -0.90 & -2.71 & -4.87 & -4.98 \\
$Y^{*} z=0.75$ & 0.21 & -1.46 & 1.00 & -0.91 & -6.92 & -7.00 & -4.60 \\
$Y^{*} z=0.5$ & 0.14 & -2.27 & 1.00 & -0.91 & -8.90 & -8.43 & -4.29 \\
\hline
\end{tabular}


of income. Consequently, the turning point decreases. The second effect dominates the first and hence the sign of this elasticity is negative. ${ }^{18}$

The third column $(A)$ and the fourth column $(\rho)$ are in line with the analytical results obtained from the special case investigated in section 4.2. The fifth $(\alpha)$ and sixth column $(\beta)$ contain negative values. Increasing either $\alpha$ or $\beta$ increases the degree of IRS in abatement, which has a strong negative impact on the turning point. ${ }^{19}$ Finally, the last column $(z)$ shows that an increase in $z$ has a strong negative impact on $Y^{*}$.

\subsubsection{The cost effectiveness of public policies}

So far we have considered first-best policies in an unconstrained welfaremaximising setting. We now turn to a cost-effectiveness analysis. It is argued that there is a maximum level of pollution, which should not be exceeded. This threshold is determined outside the economic model under study and might be the result of ecological considerations; it is not determined by costbenefit analysis. Moreover, to simplify matters, it is assumed that the regulator has only one policy instrument available in order to cap pollution. Specifically, this objective can be achieved by either implementing a tax on polluting consumption or a subsidy on environmental effort. This analysis aims to shed light on the following question: Is it optimal to primarily avoid pollution by taxing consumption, or is it instead optimal to primarily correct the problem of pollution by subsidising abatement activities? ${ }^{20}$

As a first step in trying to answer this question, we conduct the following policy experiment. The social planner implements the second-best policy (either $\tau_{C}$ or $\tau_{E}$ ) taking the optimal behaviour of the private sector into account such that the constraint $P \leq P_{\max }$ holds. Implementing a second-best consumption tax while setting the subsidy on environmental effort equal to zero is labelled a $\tau_{C}$-regime. The reverse situation is labelled a $\tau_{E}$-regime. Figure 2 illustrates the result of this policy experiment based on the baseline set of parameters (Table II) and assuming that $K_{0}=70, z=0.8$ and $P_{\max }=0.6$. Figure 2(a) shows the resulting EKC under both a $\tau_{C}$-regime and a $\tau_{E}$-regime. Figure $2(\mathrm{~b})$ shows the respective pollution paths along the time dimension. Note that the dashed segments of the respective EKCs are not realised. At those points in time where $P \leq P_{\max }$ becomes binding, the policy instruments are adjusted such that pollution remains below $P_{\max }$.

Compared to the $\tau_{C}$-regime the $\tau_{E}$-regime leads to a welfare gain which is equivalent to a permanent increase in consumption of $3.6 \%$. This is a nonnegligible number. To understand this result two points should be noted: First, Figure 2(b) shows that pollution is higher at each point in time under the $\tau_{E^{-}}$-regime, which affects welfare negatively. Second, however, the $\tau_{E^{-}}$ regime leads to a higher level of consumption than the $\tau_{C}$-regime (not 
shown), which is reasonable since the $\tau_{C}$-regime aims at discouraging polluting consumption. For the underlying set of parameters the second effect dominates. It is clear that this result is especially sensitive with respect to the parameter capturing the preference for a clean environment. Since the $\tau_{C^{-}}$ regime leads to both lower $P$ and $C$, whereas the $\tau_{E}$-regime is associated with higher $P$ and $C$, an increase in $z$ reduces or may even reverse the advantageousness of the $\tau_{E}$-regime. For instance, for $z=0.82$ the advantage of the $\tau_{E}$-regime vis-à-vis the $\tau_{C}$-regime is reduced to a welfare gain equivalent to a permanent increase in consumption of $1.7 \% .^{21}$

\section{N-shaped Pollution-income Relation}

A number of empirical studies argue that the PIR is N-shaped, at least for some pollutants (Grossman and Krueger 1995, section IV; Lieb 2003). This is important because, in this case, pollution eventually increases with income.

The model under study provides a potential explanation for this phenomenon. Imagine the economy develops at first along the upward sloping branch of the EKC resulting from the market economy (see Figure 3). At some point in time, policy instruments are implemented and pollution diminishes. In the model, the economy jumps to the centralised EKC; in reality this process is distributed over time. Provided that the economy is still below $Y^{*}$ of the centralised solution, pollution starts to increase again. This produces an N-shaped PIR resulting from the interplay of public policy and the intrinsic properties of the model. Note that this explanation implies in fact an M-shaped PIR. As soon as the peak of pollution (on the centralised EKC) is reached, pollution starts to decline.

Future empirical research aimed at explaining such a pattern should take this possibility into account. This explanation implies that the first downward movement is policy induced, i.e. it should succeed the implementation of environmental regulations aimed at a reduction of pollution. The subsequent

(a)

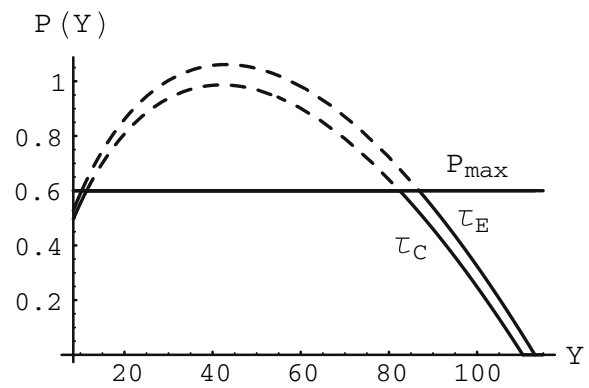

(b)

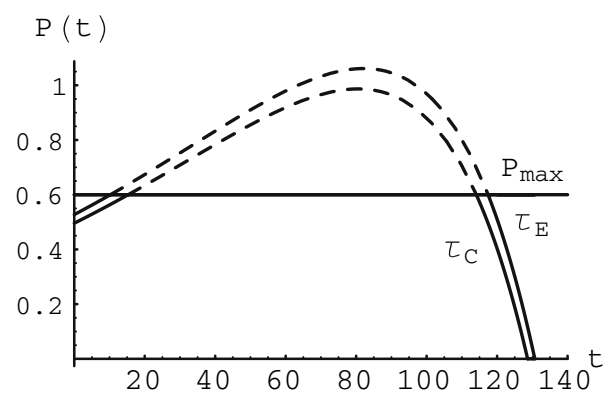

Figure 2. Comparative policy analysis. 


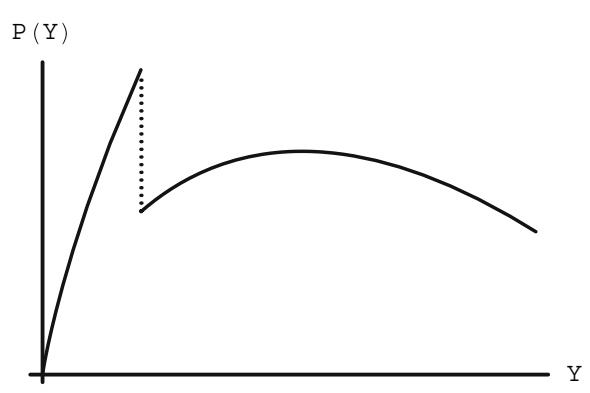

Figure 3. M-shaped PIR.

increase in pollution is then simply due to the fact that growth might be accompanied by a rise in pollution. Moreover, an $\mathrm{N}$-shaped pattern can result provided that there are less than IRS in abatement. Finally, Giles and Mosk (2003) find indeed an M-shaped EKC pattern using long-run data on methane emissions for New Zealand.

\section{Other Empirical Regularities}

A dynamic EKC model should not only reproduce an inverted U-shaped PIR. It should also be compatible with the remaining empirical regularities on economic growth and the environment. These have been reported by Brock and Taylor (2004) based on US data for 1950-2001: First, the emission intensities $(P / Y$ in our notation) for most pollutants are declining over time. Second, despite the fact that emission intensities decline, the emission levels $(P)$ continue to increase for a certain period of time. Third, abatement costs relative to GDP $(E / Y)$ are roughly constant.

The above model is compatible with these empirical regularities. Figure 4(a) shows that the emission intensity $(P / Y)$ is declining over time and that the pollution level $(P)$ continues to increase for a certain period of time although pollution intensity is falling. ${ }^{22}$ Figure $4(\mathrm{~b})$ illustrates that abatement expenditures relative to GDP $(E / Y)$ are constant over time.

The model, being an augmented AK growth model, is compatible with most of the Kaldor (1961) facts: the growth rate of per capita output, the capital-output ratio and the real rate of return on capital are constant.

\section{Summary and Conclusions}

We have set up a simple dynamic EKC model with multiple market failures resulting from external effects associated with polluting consumption and environmental effort. The model has been used to investigate the determinants of the level of income at which pollution starts to decline (turning point) as well 
(a)

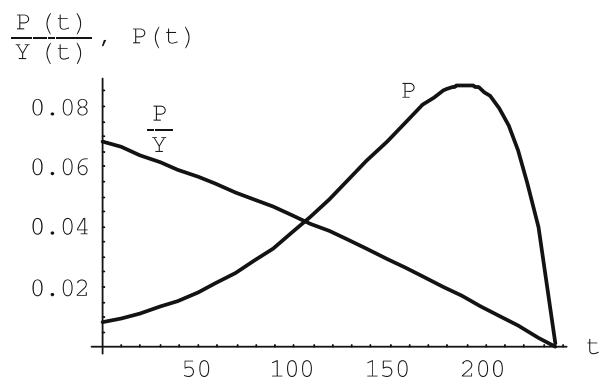

(b)

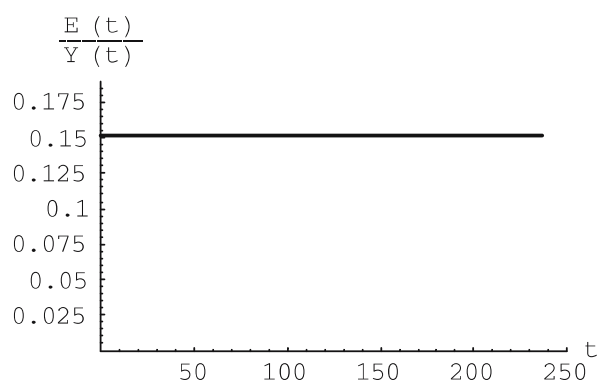

Figure 4. Comparison to empirical regularities.

as the relative effectiveness of public policy measures aimed at a reduction of the environmental burden. The main results can be summarised as follows:

(1) The turning point in the first-best solution is most strongly affected by the degree of IRS in abatement and the preference for a clean environment. In addition, in the decentralised economy, the magnitude of external effects associated with polluting consumption and environmental effort also has a substantial impact. This result indicates the importance of public policy measures for controlling pollution.

(2) For the case policy aims at a cap on pollution, which is determined by ecological factors without a cost-benefit analysis, we investigate the following question: Is it optimal to primarily avoid pollution by taxing consumption or is it instead optimal to primarily correct the problem of pollution by subsidising abatement activities? Provided that only one policy instrument is available, it turns out that a subsidy on environmental effort should be preferred vis-à-vis a tax on polluting consumption, unless the preference for a clean environment is relatively high.

(3) It has been shown that an N-shaped PIR, observable for some specific pollutants, can potentially be explained from the interaction of public policy measures and the intrinsic properties of the model. Although we do not consider this explanation to be valid in general, we think that this kind of reasoning should be taken into account in future empirical research aimed at explaining this pattern.

(4) In addition to the empirical EKC hypothesis, the dynamic EKC model under study is compatible with the remaining empirical regularities associated with economic growth and the environment (Brock and Taylor 2004). Moreover, the model is also compatible with most of the stylised facts on economic growth due to Kaldor (1961). 


\section{Acknowledgements}

\section{We are grateful for helpful comments and suggestions from Geir Asheim, Lucas Bretschger, Karen Pittel, Sjak Smulders, Scott Taylor and three anonymous referees.}

\section{Notes}

1. Lieb (2002) shows that the normality of environmental quality is a necessary condition for the existence of an EKC.

2. There are other general growth models with pollution and external effects (e.g. Smulders and Gradus 1996).

3. We do not restrict the cross derivatives at this stage.

4. More frequently, pollution is modelled as a by-product of production (e.g. Xepapadeas 2006). There are, however, other theoretical studies, beside AL (2001), which assume that consumption generates pollution (e.g. John and Pecchenino 1994).

5. Since we are interested in an EKC, we consider "interior solutions" where $B<G$. In addition, the transversality condition $\lim _{t \rightarrow \infty} e^{-\rho t} \lambda K=0$ must hold. We assume that the necessary conditions are also sufficient for a maximum of the utility functional.

6. Once again, the transversality condition $\lim _{t \rightarrow \infty} e^{-\rho t} \lambda K=0$ must hold and we assume that the necessary conditions are also sufficient.

7. Notice that $U_{C}+U_{P}\left(P_{C}+P_{\bar{C}}\right)=\lambda>0$.

8. An appendix, available upon request, shows that the parameterised Hamiltonian functions are concave such that the necessary conditions are also sufficient for a maximum of the utility functional.

9. The utility function requires $C-z P \geq 0$. For $z \leq 1$ this restriction is automatically satisfied since $C$ is gross pollution and $P$ is net pollution. Moreover, the utility function implies $U_{C P}=\frac{1}{(C-z P)^{2}}>0$, which might appear counterintuitive. A rise in $P$ has the same effect as a reduction in $C$, hence $U_{C}$ increases with $P$. According to Michel and Rotillon (1995) $U_{C P}>0$ can be interpreted as a compensation effect.

10. In a more general version of the AL (2001) model Plassmann and Khanna (2004, p. 16) show that "for non-constant returns to scale in gross pollution, a sufficient condition for pollution to decline is rather that the returns to scale in abatement exceed the returns to scale in gross pollution."

11. This is basically the solution for $Y^{*}$ one would obtain from the AL (2001) model.

12. To simplify notation, we define $\gamma=\alpha+\beta+\eta$.

13. A similar reasoning would apply to the case $\alpha \neq \beta+\eta$.

14. Since we are considering the centralised solution with $z=1, \frac{\partial Y^{*}}{\partial \eta}=\frac{\partial Y^{*}}{\partial \beta}$.

15. In this case, the relevant range of consumption is $0<C<1$. Within this range an increase in $\alpha$ lowers, ceteris paribus, abatement output. As a result, the maximum level of pollution occurs at a higher $C$-level. With $\alpha=\beta+\eta$ the rate of consumption is independent of $\alpha$ and hence a higher $C$-level implies a higher $Y^{*}$.

16. The tax rates imposed are specified as $\tau_{C}=\theta_{C} \tau_{C}^{*}$ and $\tau_{E}=\theta_{E} \tau_{E}^{*}$, where $\tau_{C}^{*}>0$ and $\tau_{E}^{*}<0$ are optimal taxes (section 3.3); $\theta_{C} \geq 0$ and $\theta_{E} \geq 0$ indicate the extent of tax implementation.

17. The elasticities are based on an $10 \%$ increase of the parameter under consideration.

18. The results are nearly identical for the unregulated and the imperfectly regulated economy. This is due to the fact that the IRS argument does not depend on the degree of regulation. 
19. As for the analytical solution the impact of $\delta$ is zero.

20. Ecologists usually argue in favour of the first strategy, whereas economists are more likely to prefer a combined strategy.

21. Although the welfare gain shrinks as $z$ converges to unity, the $\tau_{E}$-regime is preferable as long as $z<1$.

22. Figure 4 is based on the centralised solution with $z=1$ and the baseline set of parameters.

\section{References}

Anderson, D. and W. Cavendish (2001), 'Dynamic Simulation and Environmental Policy Analysis: Beyond Comparative Statics and the Environmental Kuznets Curve', Oxford Economic Papers 53(4), 721-746.

Andreoni, J. and A. Levinson (2001), 'The Simple Analytics of the Environmental Kuznets Curve', Journal of Public Economics 80(2), 269-286.

Brock, W.A. and M.S. Taylor, (2004), 'The Green Solow Model', NBER Working Paper Series, No. 10557.

Brunner, M. and H. Strulik (2002), 'Solution of Perfect Foresight Saddlepoint Problems: A Simple Method and Applications', Journal of Economic Dynamics and Control 26(5), 737 753.

Chimeli, A. B. and J. B. Braden, (2002), 'The Environmental Kuznets Curve and Optimal Growth', Working Paper, Columbia University.

Dasgupta, S., B. Laplante, H. Wang and D. Wheeler (2002), 'Confronting the Environmental Kuznets Curve', Journal of Economic Perspectives 16(1), 147-168.

Egli, H. (2005), 'A New Approach to Pollution Modelling in Models of the Environmental Kuznets Curve', Swiss Journal of Economics and Statistics 141(3), 459-473.

Eicher, Th. S. and St. J. Turnovsky (2001), 'Transitional Dynamics in a Two Sector Non-Scale Growth Model', Journal of Economic Dynamics and Control 25(1-2), 85-113.

Giles, D.E.A. and C. Mosk, (2003), 'Ruminant Eructation and a Long-Run Environmental Kuznets' Curve for Enteric Methane in New Zealand: Conventional and Fuzzy Regression Analysis', Department of Economics, University of Victoria, Econometrics Working Paper, EWP0306.

Grossman, G. M. and A. B. Krueger (1993), 'Environmental Impacts of a North American Free Trade Agreement', in P. M.Garber, ed., The Mexico-U.S. Free Trade Agreement. Cambridge and London: MIT Press.

Grossman, G. M. and A. B. Krueger (1995), 'Economic Growth and the Environment', Quarterly Journal of Economics 110(2), 353-377.

John, A. A. and R. A. Pecchenino (1994), 'An Overlapping Generations Model of Growth and the Environment', The Economic Journal 104(427), 1393-1410.

Kaldor, N. (1961), 'Capital Accumulation and Economic Growth', in F. A.Lutz and D. C.Hague, eds., The Theory of Capital. Proceedings of a Conference held by the International Economic Association. London: Macmillan.

Lieb, Ch. M. (2002), 'The Environmental Kuznets Curve and Satiation: A Simple Static Model', Environment and Development Economics 7(3), 429-448.

Lieb, Ch. M., (2003), 'The Environmental Kuznets Curve. A Survey of the Empirical Evidence and of Possible Causes', University of Heidelberg, Department of Economics, Discussion Paper Series, No. 391. 
Lieb, Ch. M. (2004), 'The Environmental Kuznets Curve and Flow versus Stock Pollution: The Neglect of Future Damages', Environmental and Resource Economics 29(4), 483-506. Maradan, D. and A. Vassiliev (2005), 'Marginal Costs of Carbon Dioxide Abatement: Empirical Evidence from Cross-Country Analysis', Swiss Journal of Economics and Statistics 141(3), 377-410.

Michel, Ph., (1993), 'Pollution and Growth towards the Ecological Paradise', Fondazione Eni Enrico Mattei, Nota di Lavoro 80.93.

Michel, Ph. and G. Rotillon (1995), 'Disutility of Pollution and Endogenous Growth', Environmental and Resource Economics 6(3), 279-300.

Ortigueira, S. and M. S. Santos (1997), 'On the Speed of Convergence in Endogenous Growth Models', American Economic Review 87(3), 383-399.

Plassmann, F. and N. Khanna, (2004), 'A Note on "The Simple Analytics of the Environmental Kuznets Curve", Working Paper 0314, Binghamton University, Department of Economics (forthcoming in Environment and Development Economics).

Selden, Th. M. and D. Song (1995), 'Neoclassical Growth, the J Curve for Abatement, and the Inverted U Curve for Pollution', Journal of Environmental Economics and Management 29(2), 162-168.

Smulders, S., L. Bretschger and H. Egli, (2005), 'Economic Growth and the Diffusion of Clean Technologies: Explaining Environmental Kuznets Curves', Economics Working Paper Series 05/42, ETH Zurich.

Smulders, S. and R. Gradus (1996), 'Pollution Abatement and Long-Term Growth', European Journal of Political Economy 12(3), 505-532.

Stern, D. I. (2004), 'The Rise and Fall of the Environmental Kuznets Curve', World Development 32(8), 1419-1439.

Stokey, N. L. (1998), 'Are there Limits to Growth?', International Economic Review 39(1), 131.

Xepapadeas, A., (1994), 'Long-Run Growth, Environmental Pollution, and Increasing Returns', Fondazione Eni Enrico Mattei, Nota di Lavoro 67.94.

Xepapadeas, A., (2006), 'Economic Growth and the Environment', in K.-G. Mäler and J. Vincent, eds., Handbook of Environmental Economics, Vol. 3, Chapter 23, North-Holland: Elsevier Publishers. 